Reihe Rechtswissenschaft

Band 199 


\title{
Das Verhältnis \\ von Steuerstraf- und Besteuerungsverfahren
}

\begin{abstract}
unter besonderer Berücksichtigung der Ursächlichkeit des Besteuerungsverfahrens für Beweisverwertungsverbote im Steuerstrafrecht
\end{abstract}

\author{
Judith Wenzel
}

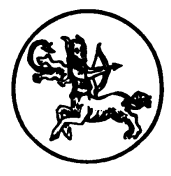

Centaurus Verlag \& Media UG 2003 
Die Autorin, geb. 1972 in Waldsassen, studierte Rechtswissenschaft an der Universität Regensburg und promovierte 2003 an der Universität Bayreuth. Nach ihrem Berufseinstieg bei einer 'Big Five'-Wirtschaftsprüfungsgesellschaft ist sie heute als Unternehmensjuristin bei der Bayerischen Landesbank tätig.

Die Deutsche Bibliothek - CIP-Einheitsaufnahme

\section{Wenzel, Judith:}

Das Verhältnis von Steuerstraf- und Besteuerungsverfahren unter besonderer Berücksichtigung der Ursächlichkeit des Besteuerungsverfahrens für Beweisverwertungsverbote im Steuerstrafrecht / Judith Wenzel. - Herbolzheim : Centaurus-Verl., 2003

(Reihe Rechtswissenschaft ; Bd. 199)

Zugl.: Bayreuth, Univ., Diss., 2003

ISBN 978-3-8255-0454-0 ISBN 978-3-86226-324-0 (eBook)

DOI 10.1007/978-3-86226-324-0

\section{ISSN 0177-2805}

Alle Rechte, insbesondere das Recht der Vervielfältigung und Verbreitung sowie der Übersetzung, vorbehalten. Kein Teil des Werkes darf in irgendeiner Form (durch Fotokopie, Mikrofilm oder ein anderes Verfahren) ohne schriftliche Genehmigung des Verlages reproduziert oder unter Verwendung elektronischer Systeme verarbeitet, vervielfältigt oder verbreitet werden.

(c) CENTAURUS Verlags-GmbH \& Co. KG, Herbolzheim 2003

Satz: Vorlage der Autorin

Umschlaggestaltung: DTP-Studio, Antje Walter, Hinterzarten 
Meiner Mutter zum Dank, meinem Vater zum Gedenken 


\section{Vorwort}

Die Rechts- und Wirtschaftswissenschaftliche Fakultät der Universität Bayreuth hat die vorliegende Arbeit 2003 als Dissertation angenommen.

Mein herzlicher Dank gilt insoweit Herrn Prof. Dr. Gerhard Dannecker für die wissenschaftliche Betreuung und die Erstellung des Erstgutachtens. Herrn Prof. Dr. Roland Schmitz schulde ich Dank für die Zweitkorrektur.

Der Friedrich-Ebert-Stiftung danke ich für das gewährte Graduiertenstipendium in einer wichtigen Phase der Promotion, ohne das die Dissertation nicht in dieser Form hätte zustande kommen können.

Besonderer Dank gilt schließlich meiner Mutter Frau Christa Wenzel sowie meinem lieben Freund Herrn Glen May, deren persönlicher Beistand, Rat und Humor mir Rückhalt gegeben haben und mich zugleich immer wieder den nötigen Abstand gewinnen ließen.

München, im Juli 2003

Judith Wenzel 


\section{Inhaltsverzeichnis}

Abkürzungsverzeichnis....................................................... XIX

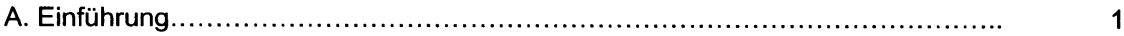

B. Doppelfunktion und Doppelzuständigkeit als Konfliktursachen.................... 5

I. Organisation der Verfolgung von Steuerstraftaten.............................. 5

1. Organisatorische Gemengelage .......................................... 7

a. Verhältnis Staatsanwaltschaft - Finanzbehörden........................ 7

(1) Selbständige Ermittlungskompetenz............................. 7

(2) Unselbständige Ermittlungskompetenz........................... 9

b. Aufgabenverteilung innerhalb der Finanzbehörden..................... 10

(1) Numerus clausus der Ermittlungsbehörden......................... 10

(2) Doppelfunktion des Finanzamts.................................. 11

(3) Organisation der Finanzämter...................................... 13

(a) Bußgeld- und Strafsachenstellen................................. 13

(b) Steuerfahndung.................................................. 15

2. Weisungs- und Eingriffsmöglichkeiten................................... 17

II. Konfliktpotential.............................................................. 19

1. Wechsel der Verfahrensart............................................... 19

2. Besorgnis der Befangenheit............................................. 20

3. Kontrolldefizit.......................................................... 22

4. Gewaltenverschränkung.................................................. 23

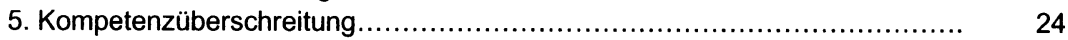

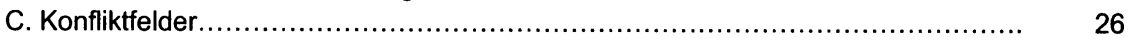

I. Belehrungs- und Aufklärungspflichten im Steuerstrafverfahren................... 26

1. Unzulässige Erzwingung von Mitwirkungshandlungen......................... 26

a. Allgemeines.......................................................... 26

(1) Rechtshistorische Entwicklung des nemo tenetur-Grundsatzes......... 27

(2) Rechtsgrundlagen des nemo tenetur-Grundsatzes.................... 28

(a) Abschaffung von Folter und Inquisition.......................... 28

(b) Unschuldsvermutung.......................................... 29

(c) Art. 6 I EMRK und Art. 14 III g des Internationalen Pakts über staatsbürgerliche und politische Rechte.......................... 29

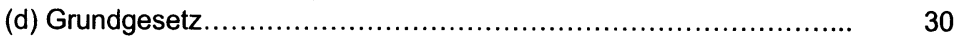

(3) Geschützter Personenkreis........................................ 31

(a) Der Beschuldigte.............................................. 32

(aa) Allgemeines................................................ $\quad 32$

(bb) Teilweises Schweigen....................................... 33

(cc) Wahrheitspflicht.......................................... 34

(dd) Sonstige Pflichten......................................... 36

(b) Der Zeuge........................................................ 36

(4) Schranken.................................................... 37

b. Bedeutung des nemo tenetur-Grundsatzes im Steuerstrafrecht............ 37

(1) $\S 393$ । $1 \mathrm{AO}$ als Widerspruch in sich............................. 37

(a) Verfassungskonforme Auslegungsmöglichkeiten.................. 39 


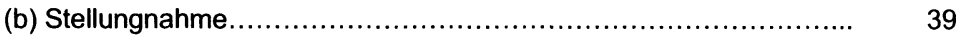

(2) Belehrung nach $\S 393$ । 4 AO...................................... 41

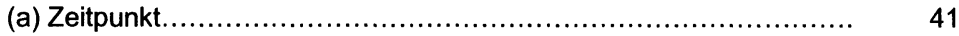

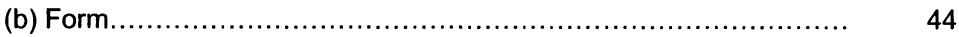

(c) Belehrungsempfänger........................................... 46

(d) Rechtsfolgen einer unterlassenen bzw. unzureichenden Belehrung $\quad 47$

(aa) Rechtsfolgen im Strafrecht.................................... 47

(bb) Rechtsfolgen im Steuerrecht............................... 49

(cc) Rechtsfolgen unzureichender Belehrungen...................... 50

(3) Reichweite im Einzelnen......................................... 50

(a) Steuerrechtliche Erklärungspflichten............................. 50

(aa) Erklärungszeitraum....................................... 51

(bb) Folgezeiträume.......................................... 52

(aaa) Allgemeines............................................. 52

(bbb) Handlungsalternativen.................................. 54

$(\alpha)$ Abgabe weiterer unrichtiger Erklärungen.............. 54

( $\beta$ ) Abgabe einer korrekten Steuererklärung in
Verbindung mit einer Selbstanzeige.................... 54

$(\chi)$ Unterlassen weiterer Steuererklärungen............... $\quad 55$

(ccc) Berücksichtigung des Selbstbelastungsverbots............ 56

$(\alpha)$ Wertungen im allgemeinen Strafrecht................ 56

( $\beta$ ) Begehungs- und Unterlassungsdelikte................ 57

( $\chi$ ) Nichtabgabe von Steuererklärungen als
Zumutbarkeitsproblem................................ 59

( $\delta$ ) Rechtsauffassung des Bundesverfassungsgerichts.... 60

(ddd) Problematik der Verdeckung einer weiteren

Steuerstraftat.............................................. 61

( $\alpha$ ) Beschluss des HansOLG Hamburg vom 7. Mai $1996 \quad 62$

( $\beta$ ) Freistellung als ultima ratio............................. 63

$(\chi)$ Eigenverursachung der „Zwangslage“................. 63

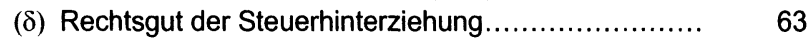

$(\alpha \alpha)$ Meinungsstand .................................. 64

$(\alpha \alpha \alpha)$ Mitteilungspflichten.......................... 64

( $\beta \beta \beta)$ Äußerer Bestand der Steueransprüche.... 65

$(\chi \chi \chi)$ Vermögensinteressen des Staates - $\quad 65$

Steueraufkommen.

$(\delta \delta \delta)$ Steuergerechtigkeit........................ 66

( $\beta \beta)$ Stellungnahme.................................. 67

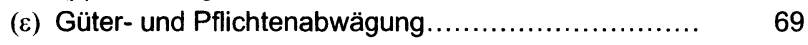

$(\alpha \alpha)$ Nichterklärung als ultima ratio.................... 69

$(\beta \beta)$ Weiteres Tatunrecht.............................. 69

$(\chi \chi)$ Schutz der Fiskalinteressen..................... 71

( $\delta \delta)$ Kompensation auf Ebene der Beweisverwertung $\quad 71$

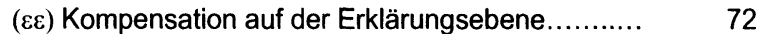

(b) Sonstige steuerrechtliche Pflichten................................. 73

c. Mittelbarer Zwang durch Gefahr höherer Schätzungen................... $\quad 75$ 
(1) Schätzungen im Steuerrecht..................................... 75

(2) Schätzungen als „Druckmittel“.................................... 76

(3) Schätzungen als unzulässiger Zwang i.S.d. § 393 । 2 AO.............. 77

(a) Problemstellung.................................................... 77

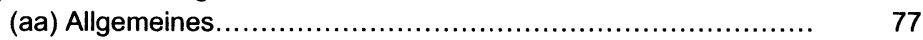

(bb) Zwang durch Schätzungen................................. 78

(aaa) Strafschätzungen....................................... 78

(bbb) Nachteilige Schätzungen............................... 78

(cc) Stellungnahme............................................ 80

(b) Beweisverwertung........................................... 83

(c) Weitere Lösungsansätze..................................... 84

(aa) Zeitliche Entzerrung......................................... 84

(bb) Einschränkung des Schätzungsermessens...................... 86

(cc) Völlige Aufhebung der Mitwirkungspflicht...................... 87

(4) Strafrechtliche Verwertbarkeit von Schätzungen....................... 88

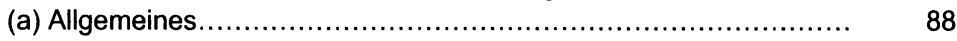

(b) Beurteilung einzelner Schätzungsverfahren........................ 91

d. Umgehung durch Mitwirkungsverlangen gegenüber Dritten.................. 94

(1) Begriff des nicht am Besteuerungsverfahren beteiligten Dritten......... 94

(2) Mitwirkungsverpflichtung des Dritten................................. 95

(a) Gleichgestellte und außenstehende Auskunftspersonen............. 95

(b) Auskunfts- und Mitwirkungsverweigerungsrechte.................... 96

(3) Zwangsweise Durchsetzung......................................... 98

(4) Strafrechtliche Mitwirkungsverpflichtungen......................... 101

(5) Umgehung......................................................... 101

(a) Rangfolge bei der Befragung Steuerpflichtiger................... 101

(b) Auswirkungen auf das Steuerstrafverfahren...................... 105

2. Nemo tenetur-Grundsatz und Beschuldigtenvernehmung..................... 105

a. Der Anfangsverdacht................................................. 105

(1) Prüfungskriterien................................................ 106

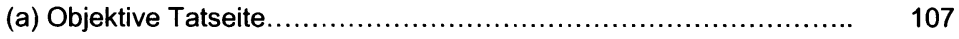

(b) Subjektive Tatseite........................................... 108

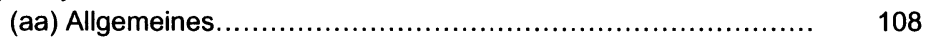

(bb) Ausprägung im Steuerstrafrecht........................... 109

(cc) Abgrenzung bedingter Vorsatz - bewusste Fahrlässigkeit...... 110

(dd) Irrtümer................................................ 111

(c) Missbrauch rechtlicher Gestaltungsmöglichkeiten................... 112

(aa) Verschleierungsabsicht..................................... 112

(bb) Bestimmtheitsgrundsatz..................................... 113

(d) Abweichen von der höchstrichterlichen Rechtsprechung............ 114

(aa) Rechtsprechung des Reichsgericht......................... 115

(bb) Rechtsprechung des Bundesgerichtshofs.................... $\quad 115$

(cc) Kritik..................................................... 116

(aaa) Bindung an das Gesetz................................. $\quad 116$

(bbb) Dokumentationspflichten.................................. 117

(ccc) Problematik des Empfängerhorizonts................... 117 
(2) Der Anfangsverdacht beim Betriebsprüfer................................. 119

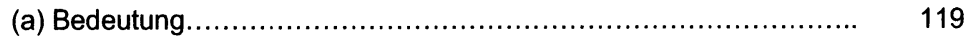

(b) Verdachtsauslösende Indizien......................................... 121

(c) Einbeziehung der Bußgeld- und Strafsachenstelle..................... 123

(aa) Ministerialerlasse der Länder.......................................... 123

(bb) Funktion und Rechtsqualität von Erlassen der

Verwaltungsbehörden.......................................... 126

(aaa) Typologie.................................................... 126

(bbb) Bindungswirkung........................................... 127

(ccc) Einordnung vorstehender Erlasse............................... 129

$(\alpha){ }_{n}$ Hartmann-Erlass“........................................ 129

$(\alpha \alpha)$ Normalverdacht .................................... 130

$(\beta \beta)$ Bindung im Außenverhältnis........................ 131

$(\chi \chi)$ Bindung im Innenverhältnis......................... 131

$(\alpha \alpha \alpha)$ Weisungsgebundenheit der

Staatsanwaltschaft............................ 132

$(\beta \beta \beta)$ Weisungsgebundenheit der strafrechtlich tätigen Finanzbehörden....................... 133

( $\delta \delta)$ Gebundenheit des einzelnen Beamten............ 136

$(\alpha \alpha \alpha)$ Interne Weisungen........................... 137

$(\beta \beta \beta)$ Allgemeine externe Weisungen.............. 137

$(\chi x \chi)$ Schranken...................................... 138

(ß) Ministerialerlasse ........................................ 139

$(\alpha \alpha)$ Regelungegenstand.............................. 139

$(\beta \beta)$ Zuständigkeitsverlagerung........................... 140

$(\chi \chi)$ Materielle Regelungen............................... 141

(cc) Regelungen der AStBV (Steuer).................................. 143

(aaa) Historie und Regelungsgegenstand.......................... 143

(bbb) Bindungswirkung................................................ 144

(ccc) Inhaltliche Kritik............................................. 144

(ddd) Fazit.......................................................... 145

(3) Der Anfangsverdacht der Steuerfahndung.................................. 145

(a) Doppelfunktion der Steuerfahndung..................................... 145

(b) Vorfeldermittlungen........................................................... 146

(aa) Einordnung der Vorfeldermittlungen................................ 147

(aaa) Strafrechtliche Verdachts-Vorermittlungen.................. 147

(bbb) Allgemeiner Steuersicherungsauftrag........................ 148

(bb) Anlass und Ausmaß von Vorfeldermittlungen....................... 149

(aaa) Ermittlungsanlass................................................ 149

(bbb) Aufgaben und Befugnisse.................................... 150

(cc) Abgrenzung zu Vorermittlungen....................................... 153

(4) Justiziabilität des Anfangsverdachts........................................ 155

(a) Maßstab der kriminalistischen Erfahrung................................ 155

(b) Justiziabilität von Fehlentscheidungen.................................. 155

(c) Überschreiten des Beurteilungsspielraums.......................... 156

(aa) Pflichtwidriges Einleiten.............................................. 156 
(bb) Pflichtwidriges Nicht-Einleiten................................ 157

(d) Rechtliche Würdigung....................................... 158

b. Belehrungspflicht nach $\S \S 136$ I 2, 163 a IV 2 StPO i.V.m. $\S 385$ | AO..... 160

(1) Begründung der Beschuldigteneigenschaft........................... 161

(a) Allgemeines................................................. 161

(b) Maßnahmen im Steuerstrafrecht............................... 163

(aa) Neutrale Maßnahmen...................................... 163

(bb) Strafrechtlicher Hinweis nach $\S 201$ II AO..................... 163

(cc) Abgrenzung Beschuldigtenvernehmung - informatorische

Befragung.................................................... 166

(2) Einleitung des Steuerstrafverfahrens............................... 167

(3) Rechtsfolgen unterlassener Belehrung........................... 170

(a) Ursprüngliche Meinung des Bundesgerichtshofs................... 171

(b) Kritik im Schrifttum............................................ 171

(c) Steuerstrafrechtliche Literatur.................................... 172

(d) Rechtsprechungsänderung des Bundesgerichtshofs.............. 173

(e) Auswirkungen im Steuerstrafverfahren........................... 174

(aa) Besonderes Schutzbedürfnis des Steuerpflichtigen.............. 174

(bb) Belehrungspflichten im Einzelnen................................ 175

(cc) Rechtsfolge unterlassener Belehrung....................... 176

(f) Einschränkungen............................................... 177

(aa) Widerspruchslösung.......................................... 177

(bb) Beweislast.................................................... 178

(aaa) Stattfinden der Belehrung................................ 178

(bbb) Kenntnis prozessualer Rechte.......................... $\quad 180$

(cc) Anwesenheit eines Verteidigers................................ 181

II. Einzelne Ermittlungsmaßnahmen im Steuerstrafverfahren..................... 183

1. Durchsuchungen............................................................. $\quad 184$

a. Fehler der Durchsuchungsanordnung................................ 185

(1) Vollständiges Fehlen einer richterlichen Durchsuchungsanordnung... $\quad 185$

(a) Anordnungslose Erstdurchsuchung............................ 186

(aa) Schwere des Grundrechtseingriffs und seine Folgen für den

Betroffenen.................................................. 186

(bb) Gewicht der Tat............................................ 187

(cc) Bewusster Verfahrensverstoß der Ermittler.................... 187

(dd) Rechtmäßiger hypothetischer Ermittlungsverlauf................ 188

(ee) Stellungnahme............................................... 189

(b) Wiederholende Durchsuchung ohne erneute Anordnung............ 189

(2) Irrtümliche Annahme von Gefahr im Verzug............................ 191

(a) Eilkompetenzen.............................................. 191

(b) Begriffsbestimmung........................................... 191

(c) Rechtsnatur der Eilentscheidung.................................. 192

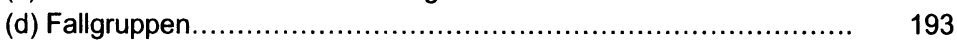

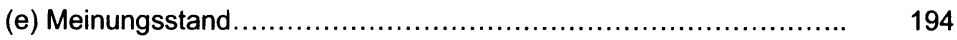

(f) Stellungnahme.............................................. 195

(3) Rechtmäßigkeit einer richterlichen Anordnung...................... 197 


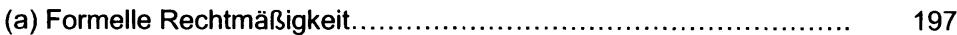

(aa) Zuständigkeit................................................. 197

(aaa) Anordnungskompetenz.............................. 197

(bbb) Antragskompetenz...................................... 197

$(\alpha)$ Allgemeines........................................ 197

( $\beta$ ) Antragskompetenz der Steuerfahndung............... 198

$(\alpha \alpha)$ Befürworter..................................... 198

$(\beta \beta)$ Kritiker...................................... 199

$(\chi \chi)$ Ergebnis...................................... 201

$(\delta \delta)$ Rechtsfolge von Verstößen...................... 202

(bb) Form........................................................ 204

(cc) Begründung und Bestimmtheit............................. 205

(aaa) Kriterien des Bundesverfassungsgerichts................. 205

(bbb) Grenzen des Bestimmtheitserfordernisses................ 207

(ccc) Stellungnahme............................................ 208

(ddd) Rechtsfolge von Verstößen.............................. 209

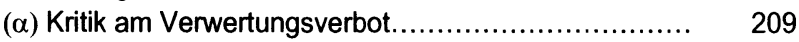

( $\beta$ ) Befürworter eines Verwertungsverbots................. 210

$(\chi)$ Stellungnahme...................................... 210

(b) Materielle Rechtmäßigkeit....................................... 211

(aa) Vorliegen eines Tatverdachts................................. 211

(aaa) Notwendiger Verdachtsgrad............................ 211

$(\alpha)$ Allgemeines Strafrecht............................. 211

( $\beta$ ) Besonderheiten im Steuerstrafrecht................... $\quad 212$

(bbb) Bankenfälle............................................. 212

$(\alpha)$ Verdacht gegenüber Bankkunden..................... 213

( $\beta$ ) Verdächtige Geschäfte.............................. $\quad 214$

$(\chi)$ Beihilfe von Bankangestellten........................ 216

( $\delta)$ Flächendeckende Ermittlungen....................... 218

(ccc) Stellungnahme.......................................... 220

(bb) Zeitliche Aspekte der Durchsuchung.......................... 223

(aaa) Verwirkungsgedanke..................................... 224

(bbb) Folgen einer Fristversäumnis............................ 225

b. Fehler bei Durchführung der Durchsuchung.............................. 226

(1) Unzuständigkeit der Finanzbehörden.................................. 226

(2) Nichtbeachtung bestimmter Verfahrensregeln........................ 226

(a) Uhrzeit und Dauer der Durchsuchung............................... 226

(aa) Durchsuchungen zur Nachtzeit............................... 226

(bb) Dauer der Durchsuchung.................................... 227

(aaa) Allgemeines.......................................... 227

(bbb) Absolute Obergrenze..................................... 228

(ccc) Würdigung........................................... 228

(ddd) Rechtsfolgen einer zu langen Sichtung.................. 229

(b) Hinzuziehung von Durchsuchungszeugen......................... 230

(aa) Allgemeines................................................... 230

(bb) Besonderheiten im Steuerstrafverfahren....................... 230 
(cc) Rechtsfolgen einer unterlassenen Zeugenhinzuziehung.........

(c) Durchsicht von Papieren....................................... 232

(aa) Zuständigkeitsregelung..................................... 232

(bb) Inhalt und Umfang.......................................... 234

(cc) Mögliche Verfahrensfehler.................................. $\quad 235$

(aaa) Sichtung von Privatpapieren.......................... $\quad 235$

(bbb) Delegation der Durchsichtsbefugnis..................... $\quad 235$

$(\alpha)$ Befürworter...................................... $\quad 235$

( $\beta$ ) Gegenansicht...................................... 236

$(\chi)$ Stellungnahme................................... 236

(dd) Rechtsfolge von Verstößen.................................. 237

(3) Überschreiten der Durchsuchungsbegrenzungen.................... 237

c. Grundsatz der Verhältnismäßigkeit.................................... 239

(1) Durchsuchungsanordnung....................................... 239

(2) Durchsuchungsvollzug............................................. 240

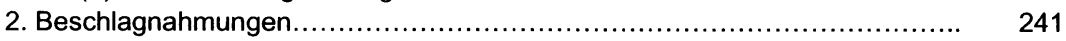

a. Parallelen zur Durchsuchung......................................... 241

b. Beschlagnahmeverbote............................................... 243

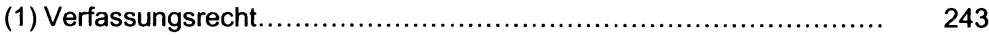

(2) Berufsgruppenspezifische Verbote................................... 244

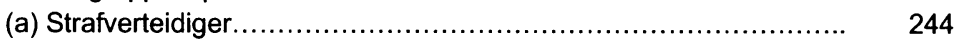

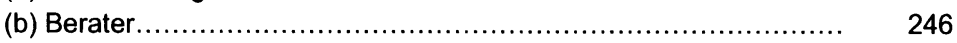

(aa) Geschützte Gegenstände.................................. 247

(aaa) Schriftliche Mitteilungen und Aufzeichnungen............. 247

(bbb) „Andere Gegenstände“................................ 247

$(\alpha)$ Übergabeargument................................. 248

( $\beta)$ Gewahrsamskriterium.............................. $\quad 249$

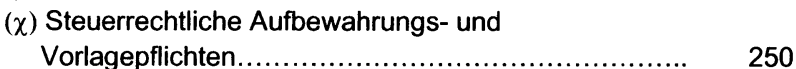

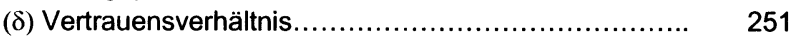

(ع) Berufstypisches Handeln des Beraters................. $\quad 252$

( $\phi)$ Zeitliche Begrenzung............................... 253

(bb) Grenzen des Beschlagnahmeverbots........................ 254

(aaa) Teilnahmeverdacht...................................... 254

(bbb) Tatwerkzeuge.......................................... 255

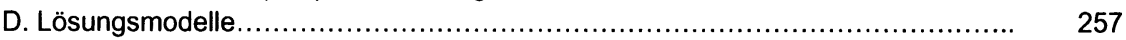

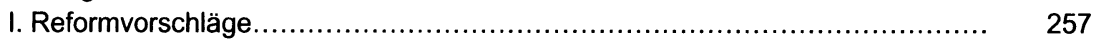

1. Funktionstrennung .................................................... 257

2. Kompetenzbeschneidung................................................ 260

3. Verfahrensaussetzung................................................... 262

a. Aussetzung des Besteuerungsverfahrens............................... 262

b. Aussetzung des Steuerstrafverfahrens................................... 264

(1) $\S 396 \mathrm{AO}$ als Rechtsgrundlage................................... 264

(2) Aussetzungspflicht............................................. 266

(a) Vorfragenkompetenz des Strafrichters.......................... 266

(b) Bindungswirkung steuerrechtlicher Entscheidungen................ 267 
(aa) Interpretationsvorrang der Finanzbehörden

(bb) Tatbestandswirkung von Verwaltungsakten........................ 269 (aaa) Wesen der Tatbestandswirkung .............................. 269 (bbb) Voraussetzungen............................................. 271

(a) Verweisung auf verwaltungsrechtliche Normen........ 271

(ß) Begründung eines Ge- oder Verbots..................... 271

(cc) Urteile der Finanzgerichte.......................................... 273

(3) Beschleunigungsgebot - Art. 6 EMRK................................... 275

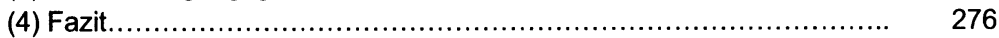

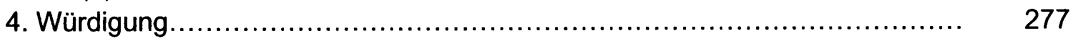

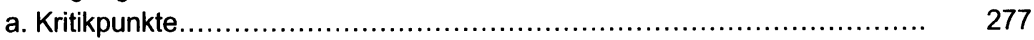

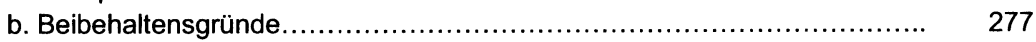

c. Verfassungsrechtliche Würdigung................................................. 279

(1) Qualifikationsdefizit..................................................... 279

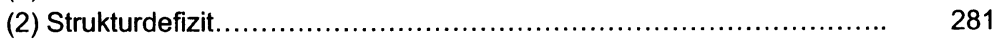

II. Das Beweisverwertungsverbot als Konfliktlösung.................................. 282

1. Einteilung und Begründung der Beweisverbote.................................... 282

a. Systematik................................................................... 282

(1) Beweiserhebungsverbote.............................................. 283

(2) Beweisverwertungsverbote............................................. 284

(a) Selbständige Beweisverwertungsverbote................................ 285

(aa) Kernbereich privater Lebensführung.............................. 285

(bb) Schlichte Privatsphäre ............................................. 286

(cc) Bereich außerhalb der Privatsphäre............................. 286

(b) Unselbständige Beweisverwertungsverbote.......................... 287

(aa) Revisionsrechtliche Theorien..................................... 287 (aaa) Allgemeines................................................ 287

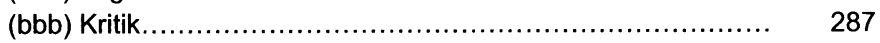

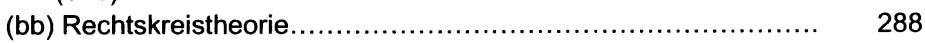

(aaa) Allgemeines............................................... 288

(bbb) Kritik ................................................................. 289

(cc) Präventionslehren................................................. 290

(aaa) Spezialprävention.............................................. 290

( ) Allgemeines......................................... 290

( $\beta$ K Kritik ................................................... 290

(bbb) Generalprävention.............................................. 291

$(\alpha)$ Allgemeines........................................... 291

( $\beta$ ) Kritik..................................................... 292

(dd) Schutzzwecklehre ............................................... 292

(aaa) Allgemeines................................................... 292

(bbb) Kritik ........................................................ 293

(ee) Abwägungslehre..................................................... 294

(aaa) Allgemeines ............................................... 294

(bbb) Kritik ........................................................ 295

(ff) Lehre von den Informationsbeherrschungsrechten................ 296

(aaa) Allgemeines .................................................. 296 
(bbb) Kritik............................................. 298

b. Würdigung.............................................................. 300

(1) Allgemeines....................................................... 300

(2) Zweck und Funktion der Beweisverwertungsverbote.................. 300

(a) Schutz der Wahrheitsfindung .................................. 301

(aa) Materielle Wahrheit....................................... 301

(bb) Prozessuale Wahrheit.......................................... 302

(b) Disziplinierung der Ermittler................................... 302

(c) Reinheit des Verfahrens....................................... 305

(d) Rechtsschutztheorien......................................... 308

(aa) Rechtsmitteltheorie......................................... 308

(bb) Schutz von Individualrechten................................ 309

c. Einbeziehung steuerstrafverfahrensrechtlicher Wertungen................. 311

(1) Bedeutung einzelner Verbotstheorien.............................. 311

(2) Bedeutung einzelner Verbotszwecke............................. 313

d. Zusammenfassende Stellungnahme...................................... 314

2. Umfang der Beweisverwertungsverbote................................... $\quad 317$

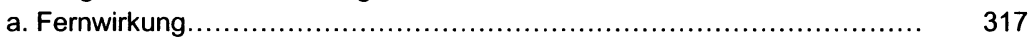

(1) Behandlung unmittelbarer Beweise................................ 317

(2) Behandlung mittelbarer Beweise ................................. 318

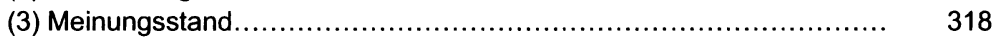

(a) Befürworter der Fernwirkung..................................... 318

(b) Kritiker der Fernwirkung........................................ 319

(4) Würdigung.......................................................... 320

(a) Intensität des Verstoßes...................................... 320

(b) Kausalität des Verstoßes....................................... 321

(c) Gesetzliche Verankerung im Insolvenzrecht........................ 321

(aa) Umsetzung des Gemeinschuldnerbeschlusses................. 321

(bb) Ausgestaltung der Fernwirkung................................ 322

(cc) Einschränkungen............................................ 325

(d) Auswirkungen im Steuerstrafverfahren.......................... 327

b. Zusammenhang zwischen dem steuerstrafrechtlichem Verwertungsverbot und dem Verwertungsverbot im Besteuerungsverfahren.

(1) Durchgriff strafprozessualer Verwertungsverbote auf das

Besteuerungsverfahren

329

(a) Steuerlicher Ansatz ............................................ 330

(b) Steuerliche Auswirkungen strafprozessualer Verwertungsverbote... 333

(aa) Allgemeines.............................................. 333

(aaa) Meinungsstand......................................... 333

(bbb) Stellungnahme...................................... 334

(bb) Fallgruppen .............................................. $\quad 335$

(aaa) Verletzung von Grundrechten............................ $\quad 335$

$(\alpha) \S 136$ a StPO ................................... 336

( $\beta$ ) Allgemeines Persönlichkeitsrecht.................... 337

$(\chi)$ Art. 13 GG....................................... 338

(bbb) Verletzung einfachen Rechts.......................... 339 
( $\alpha)$ Allgemeines.

(ß) Verletzung von $\S \S 136$ । 2, 163 a IV 2 StPO i.V.m. $\S 385$ I AO.

$(\chi)$ Verletzung von § 393 । 4 AO............................. 341

(c) Geltendmachung des Verwertungsverbots............................. 342

(2) Auswirkung steuerlicher Verwertungsverbote auf nachfolgende

Strafverfahren......

343

E. Zusammenfassung der Ergebnisse.

344

Literaturverzeichnis.

357 


\section{Abkürzungsverzeichnis}

a.A. anderer Ansicht

a.a.O. am angegebenen Ort

Abs.

Absatz

a.E.

am Ende

a.F.

AG

alte Fassung

AJCL

Amtsgericht

AK-GG

The American Journal of Comparative Law

Alternativkommentar zum Grundgesetz für die Bundesrepublik

Deutschland

AK-StGB

Alternativkommentar zum Strafgesetzbuch

AK-StPO

Alternativkommentar zur Strafprozeßordnung

Alt.

Alternative

Anm.

Anmerkung

AnwBI.

Anwaltsblatt

$\mathrm{AO}$

Abgabenordnung

AOStrafÄndG

Gesetz zur Änderung strafrechtlicher Vorschriften der

Art.

Reichsabgabenordnung und anderer Gesetze

AStBV

AT

Artikel

Anweisungen für das Straf- und Bußgeldverfahren (Steuer)

Allgemeiner Teil

\section{BayObLG}

BayObLGSt

Bayerisches Oberstes Landesgericht

Amtliche Sammlung von Entscheidungen des Bayerischen Obersten Landesgerichts in Strafsachen

BayVBI.

Bayerische Verwaltungsblätter

BayVGH

$\mathrm{BB}$

Bayerischer Verwaltungsgerichtshof

BBG

Bd.

Betriebs-Berater

Bundesbeamtengesetz

Bek.

Band

BerlinFG

Bekanntmachung

Berlinförderungsgesetz

BergPG

Beschl.

Gesetz über Bergmannsprämien

BeurkG

BFH

Beschluss

BFHE

Beurkundungsgesetz

Bundesfinanzhof

BGB

Sammlung der Entscheidungen des Bundesfinanzhofs

BGBI.

Bürgerliches Gesetzbuch

$\mathrm{BGH}$

BGHSt

$\mathrm{BGHZ}$

Bundesgesetzblatt

Bundesgerichtshof

Amtliche Sammlung von Entscheidungen des Bundesgerichtshofs in Strafsachen

Amtliche Sammlung von Entscheidungen des Bundesgerichtshofs in Zivilsachen

BImSchG Bundes-Immissionsschutzgesetz 


\begin{tabular}{|c|c|}
\hline BKGG & Bundeskindergeldgesetz \\
\hline BK-GG & Bonner Kommentar zum Grundgesetz \\
\hline BMF & Bundesministerium der Finanzen \\
\hline $\mathrm{BpO}$ & Betriebsprüfungsordnung \\
\hline BRAO & Bundesrechtsanwaltsordnung \\
\hline BR-Drs. & Drucksache des Bundesrates \\
\hline BRRG & Beamtenrechtsrahmengesetz \\
\hline BStBI. & Bundessteuerblatt \\
\hline BT-Drs. & Drucksache des Bundestages \\
\hline Buchst. & Buchstabe \\
\hline BuStra & Bußgeld- und Strafsachenstelle \\
\hline BVerfG & Bundesverfassungsgericht \\
\hline BVerfGE & $\begin{array}{l}\text { Amtliche Sammlung von Entscheidungen des } \\
\text { Bundesverfassungsgerichts }\end{array}$ \\
\hline BVerwG & Bundesverwaltungsgericht \\
\hline BVerwGE & $\begin{array}{l}\text { Amtliche Sammlung von Entscheidungen des } \\
\text { Bundesverwaltungsgerichts }\end{array}$ \\
\hline bzW. & bezlehungswelse \\
\hline ca. & circa \\
\hline cpd & conto pro diverse \\
\hline DB & Der Betrieb \\
\hline ders. & derselbe \\
\hline d.h. & das heißt \\
\hline DM & Deutsche Mark \\
\hline DNotZ & Deutsche Notar-Zeitschrift \\
\hline DONot & Dienstordnung für Notare \\
\hline DÖV & Die Öffentliche Verwaltung \\
\hline DRiG & Deutsches Richtergesetz \\
\hline DRiZ & Deutsche Richterzeitung \\
\hline Drs. & Drucksache \\
\hline DStR & Deutsches Steuerrecht \\
\hline DStZ & Deutsche Steuer-Zeitung \\
\hline DStZ A & Deutsche Steuer-Zeitung Ausgabe A \\
\hline DVBI. & Deutsche Verwaltungsblätter \\
\hline EDV & elektronische Datenverarbeitung \\
\hline EFG & Entscheidungen der Finanzgerichte \\
\hline EG & Europäische Gemeinschaften \\
\hline EGGVG & Einführungsgesetz zum Gerichtsverfassungsgesetz \\
\hline EGMR & Entscheidungen des Europäischen Gerichtshofs für Menschenrechte \\
\hline EGOWiG & Einführungsgesetz zum Gesetz über Ordnungswidrigkeiten \\
\hline Einl. & Einleitung \\
\hline EMRK & $\begin{array}{l}\text { Europäische Konvention zum Schutze der Menschenrechte und } \\
\text { Grundfreiheiten }\end{array}$ \\
\hline
\end{tabular}




$\begin{array}{ll}\text { EStDV } & \text { Einkommensteuer-Durchführungsverordnung } \\ \text { EStG } & \text { Einkommensteuergesetz } \\ \text { etc. } & \text { et cetera } \\ \text { EuGH } & \text { Gerichtshof der Europäischen Gemeinschaften } \\ \text { EuGRZ } & \text { Europäische Grundrechte Zeitschrift } \\ \text { EWR } & \text { Europäisches Weinrecht } \\ & \\ \text { FAGO } & \text { Geschäftsordnung für die Finanzämter } \\ \text { FAZ } & \text { Frankfurter Allgemeine Zeitung } \\ \text { f./ff. } & \text { folgende } \\ \text { FG } & \text { Finanzgericht } \\ \text { FGO } & \text { Finanzgerichtsordnung } \\ \text { FK-InsO } & \text { Frankfurter Kommentar zur Insolvenzordnung } \\ \text { Fn. } & \text { Fußnote } \\ \text { FR } & \text { Finanz-Rundschau } \\ \text { FS } & \text { Festschrift } \\ \text { FVG } & \text { Finanzverwaltungsgesetz } \\ & \\ \text { gem. } & \text { gemäß } \\ \text { GA } & \text { Goltdamer's Archiv für Strafrecht } \\ \text { GG } & \text { Grundgesetz } \\ \text { ggf. } & \text { gegebenenfalls } \\ \text { GMBI. } & \text { Gemeinsames Ministerialblatt des Bundesministers des Innern } \\ \text { GrS } & \text { Großer Senat } \\ \text { GS } & \text { Gedenkschrift } \\ \text { GVBI. NW } & \text { Gesetz- und Verordnungsblatt für das Land Nordrhein-Westfalen } \\ \text { GVG } & \text { Gerichtsverfassungsgesetz } \\ & \\ \text { HansOLG } & \text { Hanseatisches Oberlandesgericht } \\ \text { HFR } & \text { Höchstrichterliche Finanzrechtsprechung } \\ \text { HGB } & \text { Handelsgesetzbuch } \\ \text { HGrG } & \text { Haushaltsgrundsätzegesetz } \\ \text { H/H/Sp } & \text { Hübschmann/Hepp/Spitaler, Kommentar zur AO und FGO } \\ \text { HK-StPO } & \text { Heidelberger Kommentar zur Strafprozessordnung } \\ \text { h.M. } & \text { herrschende Meinung } \\ \text { Hrsg. } & \text { Herausgeber } \\ \text { HS } & \text { Halbsatz } \\ \text { IdW } & \text { Institut der Wirtschaftsprüfer } \\ \text { i.e. } & \text { it est } \\ \text { i.e.S. } & \text { im engeren Sinne } \\ \text { INF } & \text { Die Information über Steuer und Wirtschaft } \\ \text { InvZulG } & \text { Investitionszulagengesetz } \\ \text { i.S.d. } & \text { im Sinne der/des } \\ \text { i.V.m. } & \text { in Verbindung mit } \\ \text { i.w.S. } & \text { im weiteren Sinne } \\ & \end{array}$




\begin{tabular}{|c|c|}
\hline Jura & Juristische Ausbildung \\
\hline JR & Juristische Rundschau \\
\hline JuS & Juristische Schulung \\
\hline$J Z$ & Juristenzeitung \\
\hline JW & Juristische Wochenschrift \\
\hline Kap. & Kapitel \\
\hline KG & Kammergericht \\
\hline KK-StPO & Karlsruher Kommentar zur Strafprozeßordnung, \\
\hline KöSDI & Kölner Steuerdialog \\
\hline LG & Landgericht \\
\hline LK-StGB & Strafgesetzbuch, Leipziger Kommentar \\
\hline LR & Löwe-Rosenberg, Großkommentar zur StPO \\
\hline MDR & Monatsschrift für Deutsches Recht \\
\hline Mrd. & Milliarde \\
\hline MRK & Konvention zum Schutze der Menschenrechte und Grundfreiheiten \\
\hline $\begin{array}{l}\text { MüKo-InsO } \\
\text { m.w.N. }\end{array}$ & $\begin{array}{l}\text { Münchener Kommentar zur Insolvenzordnung } \\
\text { mit weiteren Nachweisen }\end{array}$ \\
\hline NJW & Neue Juristische Wochenschrift \\
\hline Nr. & Nummer \\
\hline NStZ & Neue Zeitschrift für Strafrecht \\
\hline NV & nicht veröffentlicht \\
\hline NVwZ & Neue Zeitschrift für Verwaltungsrecht \\
\hline NWB & Neue Wirtschaftsbriefe \\
\hline $\mathrm{NZI}$ & Neue Zeitschrift für das Recht der Insolvenz und Sanierung \\
\hline OFD & Oberfinanzdirektion \\
\hline o.g. & oben genannte/r \\
\hline OLG & Oberlandesgericht \\
\hline OVG & Oberverwaltungsgericht \\
\hline pp. & und so weiter \\
\hline PStR & Praxis Steuerstrafrecht \\
\hline RAO & Reichsabgabenordnung \\
\hline RFH & Reichsfinanzhof \\
\hline RFHE & $\begin{array}{l}\text { Amtliche Sammlung der Entscheidungen und Gutachten des } \\
\text { Reichsfinanzhofs }\end{array}$ \\
\hline RG & Reichsgericht \\
\hline RGSt & $\begin{array}{l}\text { Amtliche Sammlung von Entscheidungen des Reichsgerichts in } \\
\text { Strafsachen }\end{array}$ \\
\hline RiStBV & Richtlinien für das Strafverfahren und das Bußgeldverfahren \\
\hline
\end{tabular}


RStBI. Reichssteuerblatt

S.

s.a.

SK-StPO

Seite

Sig.

sog.

siehe auch

SparPG

Systematischer Kommentar zur Strafprozeßordnung und zum

Gerichtsverfassungsgesetz

StahllnvZulG

Sammlung

sogenannte

$\mathrm{StB}$

Spar-Prämiengesetz

Gesetz über eine Investitionszulage in der Eisen- und Stahlindustrie

StBerG

Der Steuerberater

StBerGebV

Steuerberatungsgesetz

Stbg

Steuerberatergebührenverordnung

StbJb

Die Steuerberatung

StBP

Steuerberater-Jahrbuch

StEK

Die steuerliche Betriebsprüfung

StGB

Steuererlasse in Karteiform

StPÄG

StPO

StraBu

StraFo

Strafgesetzbuch

Gesetz zur Änderung der Strafprozeßordnung und des

Gerichtsverfassungsgesetzes

Strafprozessordnung

Strafsachen- und Bußgeldstelle

StuW

Strafverteidiger Forum

StV

Steuer und Wirtschaft

StVj

StVRG

Strafverteidiger

Steuerliche Vierteljahres-Schrift

StVZO

StWa

Gesetz zur Reform des Strafverfahrensrechts

Straßenverkehrs-Zulassungs-Ordnung

Die Steuer-Warte

TA-Lärm

TA-Luft

Tz.

technische Anleitung zum Schutz gegen Lärm

technische Anleitung zur Reinhaltung der Luft

Textziffer

u.a.

unter anderem

u.ä.

und ähnliche

Urt.

Urteil

USA

United States of America

UStG

Umsatzsteuergesetz

usw.

und so weiter

u.U.

unter Umständen

v.

vom/von

v.a.

vor allem

VerfGH

Verfassungsgerichtshof

vgl.

vergleiche 


$\begin{array}{ll}\text { V-Leute } & \text { Vertrauensleute } \\ \text { V-Personen } & \text { Vertrauenspersonen } \\ \text { VN } & \text { Vereinte Nationen } \\ \text { VRS } & \text { Verkehrsrechts-Sammlung } \\ \text { VwGO } & \text { Verwaltungsgerichtsordnung } \\ \text { VwVfG } & \text { Verwaltungsverfahrensgesetz } \\ \text { VZ } & \text { Veranlagungszeitraum } \\ & \\ \text { wistra } & \text { Zeitschrift für Wirtschaft, Steuer, Strafrecht } \\ \text { WM } & \text { Wertpapier-Mitteilungen } \\ \text { WoPG } & \text { Wohnungsbau-Prämiengesetz } \\ \text { Wpg } & \text { Die Wirtschaftsprüfung } \\ \text { WpHG } & \text { Wertpapierhandelsgesetz } \\ \text { WPO } & \text { Wirtschaftsprüferordnung } \\ & \\ \text { z.B. } & \text { zum Beispiel } \\ \text { Ziff. } & \text { Ziffer } \\ \text { ZlnsO } & \text { Zeitschrift für das gesamte Insolvenzrecht } \\ \text { ZIP } & \text { Zeitschrift für Wirtschaftsrecht } \\ \text { ZPO } & \text { Zivilprozessordnung } \\ \text { ZRP } & \text { Zeitschrift für Rechtspolitik } \\ \text { ZStW } & \text { Zeitschrift für die gesamte Strafrechtswissenschaft }\end{array}$

